\title{
Construction of $\alpha-\beta$ phase junction on Bi4V2O11 via electrospinning retardation effect and its promoted photocatalytic performance
}

Chade Lv, Gang Chen, * Jingxue Sun, * Yansong Zhou

Department of Chemistry, Harbin Institute of Technology, 150001, P. R. China,

*Corresponding author: Email: gchen@hit.edu.cn, jxsun@hit.edu.cn. Fax:

(+86)-451-86413753 


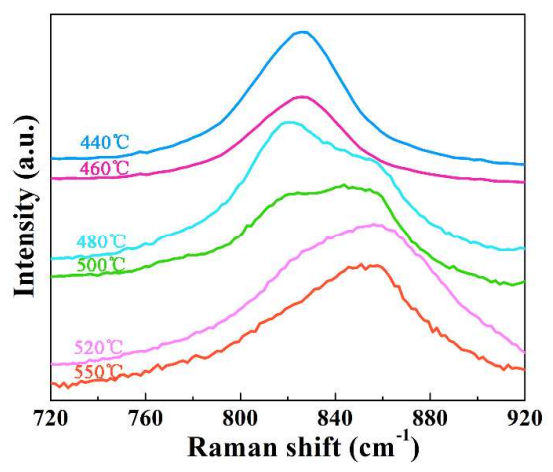

Figure S1 Raman spectra of the samples calcinated at different temperature.
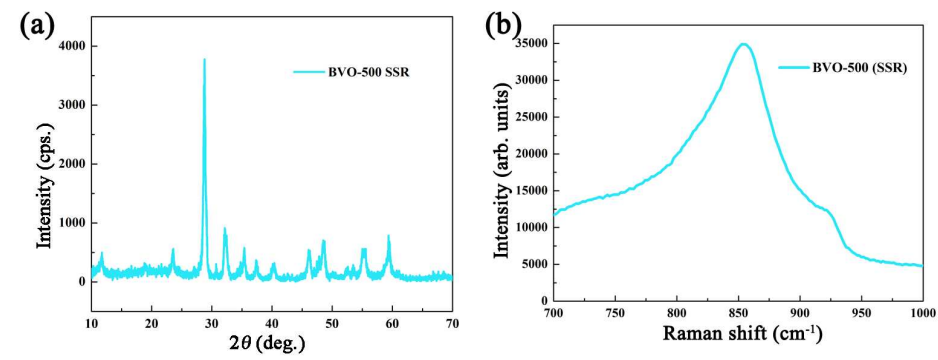

Figure S2 XRD pattern (a) and Raman spectrum (b) of BVO-500 (SSR).

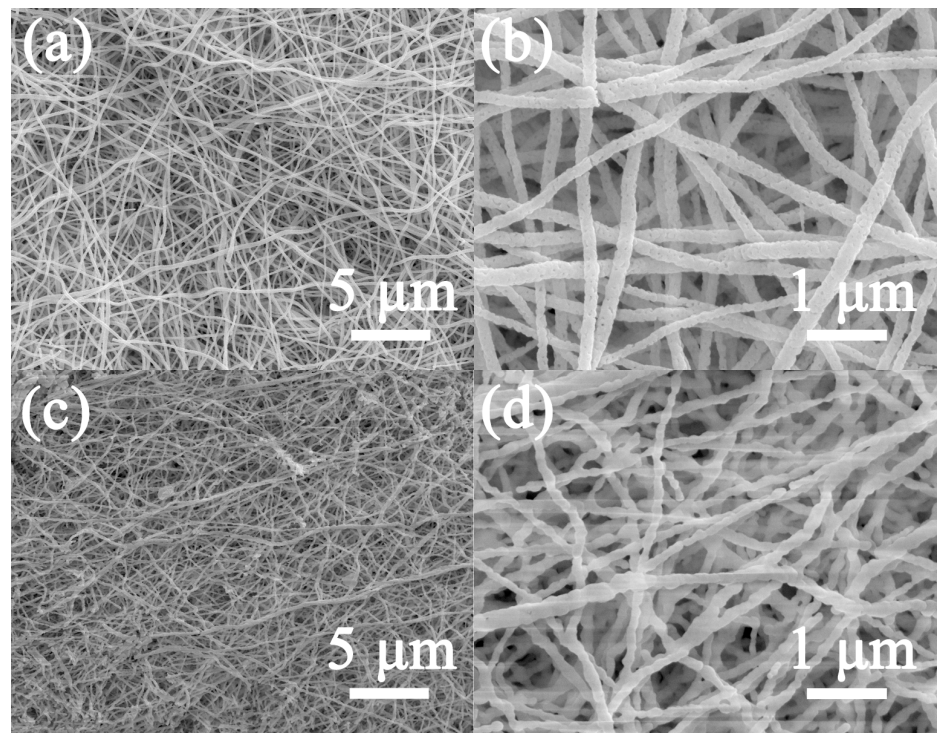

Figure S3 SEM images of BVO-440 (a, b) and BVO-550 (c,d). 


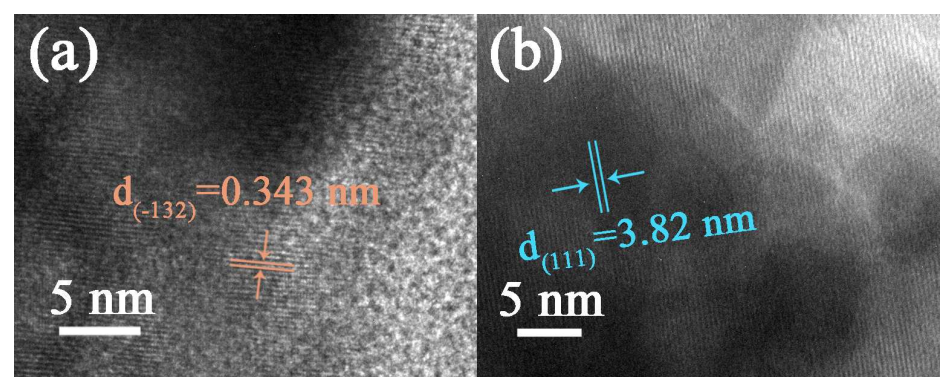

Figure S4 HRTEM images of BVO-440 (a) and BVO-550 (b)

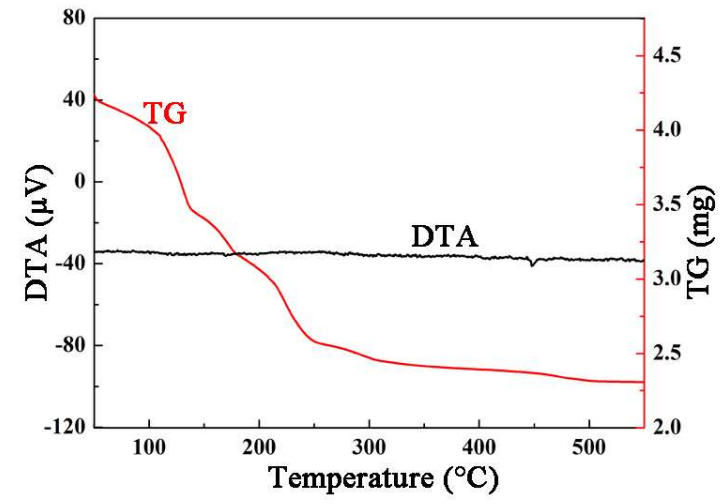

Figure S5 TG-DTA analysis of BVO-550 SSR.

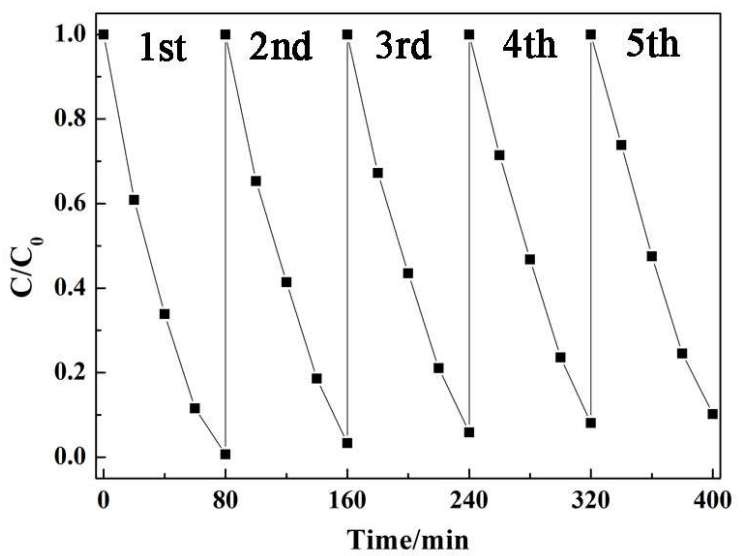

Figure S6 Photocatalytic stability measurement of BVO-500 (Cr(VI) as probe). 


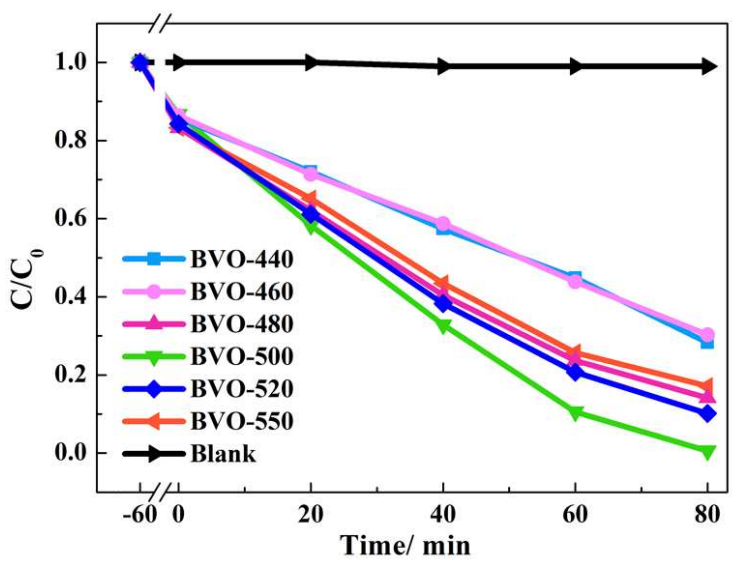

Figure S7 Dynamic curves of reduction of $\mathrm{Cr}(\mathrm{VI})$ of the samples calcinated in different temperature.
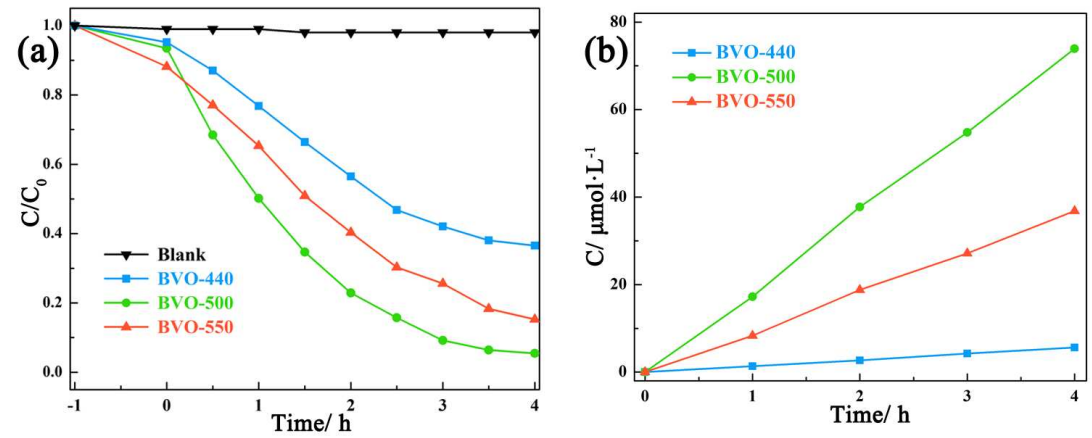

Figure S8 Dynamic curves of degradation of MB (a) and nitrogen fixation (b). 